\title{
STUDY OF NANO/MICRO AEROSOL PARTICLES MEASUREMENT FOR THE UHV SLIT VALVES
}

\author{
RONG-YUAN JOU ${ }^{1}$, JIAN-JIA QIU ${ }^{1}$, CHE-CHIN CHEN ${ }^{2}$, JAMES SU ${ }^{2} \&$ MING-HUA SHIAO ${ }^{2}$ \\ ${ }^{1}$ Department of Mechanical Design Engineering, National Formosa University, Huwei, Yunlin, Taiwan. \\ ${ }^{2}$ Instrument Technology Research Center, National Applied Research Laboratories, \\ Hsinchu Science Park, Hsinchu, Taiwan.
}

\begin{abstract}
In response to the nano-scale miniaturization trend of IC devices, advanced semiconductor processes require more stringent cleanliness. This study sets up a UHV measurement system for particles detection within a highly cleanliness testing chamber with a UHV slit valve to be test, and an experimental procedure is proposed and examined to investigate particle generation while this slit valve is in operations. Cycle numbers of 10,000, 20,000 and 40,000, respectively, are set for slit valve testing. A series of experiments are conducted to gather particles generation information and to clarify the possible causes and sources of dust particles and its concentrations and the particle sizes. The condensation particle counter (CPC) is used to measure the particle concentration and the differential mobility analyzer (DMA) is for particle sizes measurement. Besides, scanning electron microscopy (SEM) and atomic force microscope (AFM) are used to investigate the condensation behaviors on a witness wafer and the energy dispersive $\mathrm{x}$-ray spectroscopy (EDS) is used to the surface characterizations of the slit valve O-ring. In atmosphere experiments, the particle sizes and size distributions are measured by CPC and SMPS instruments and the gathered results are compared to the measured particle sizes by SEM and AFM and are used to evaluate the assumptions of particle generating sources and mechanisms. Experimental results show that the particle sizes and particle concentrations increase as the cycle numbers increases. To examine the particle generations in vacuum, the particle sizes of the deposited particles on wafer are measured by SEM and AFM. The results are compared with the SMPS measurement. Keywords: cleanliness, particle, Scanning Mobility Particle Sizer (SMPS), slit valve,
\end{abstract}

\section{INTRODUCTION}

In most vacuum systems, vacuum valves are used to be as valving for pump isolation, gas flow control, or gas admission. When selecting a vacuum valve, there are many considerations such as whether it is for shutoff or for control, the type of valve, the pressure range over which the valve is to be used, how it is to be actuated, and the seal materials. Compatibility of construction materials exposed to the process or the ability of the operating mechanism to tolerate opening against a pressure differential may also be important [1]. In view of the development of advanced semiconductor process and micro-nano systems (micro-nano systems) technology development trend, the current process requires a wafer in a process cycle after the wafer size of more than $0.2 \mu \mathrm{m}$ dust can not be more than 10 particles. Reference [2] presents a study to design and construct a measurement system and to establish its testing procedures for a developing innovative slit valve design. In their study, typical tests done for vacuum valves include: (1) vacuum performance, (2) lifetime (cycle life) and mechanical wear, (3) bake-out, (4) pressure rise tests, spectrum analysis of residual gases, (5) opening/ closing speed, (6) pressure control performance and (7) alternative sealing materials, respectively [3].

Very fast-opening gate valves offer the possibility to study fast processes in vacuum. For a dynamic vacuum primary standard where the pressure shall be reduced from $100 \mathrm{kPa}$ to 100 $\mathrm{Pa}$ in a well-defined manner within one second, a very fast-opening DN40 gate valve was developed. The opening time was investigated by a high-speed camera with 10,000 f/s for a 
differential pressure across the valve of $100 \mathrm{kPa}$ to vacuum and zero differential pressure at two different absolute pressures [4]. Trends in the semiconductor industry seen by VAT includes: (1) node size reduction, (2) low defects, (3) $450 \mathrm{~mm}$ transition and (4) lithography goes into vacuum, EUV - technology, etc. Therefore, development in the following fields necessary: (1) cleaning procedures (high purity), (2) particle performance (zero particle) [2]. Based on the past measurements, holistic understanding regarding measurement technology, influencing factors, necessary sealing technology and design methods are gained.

As to particulate matter (PM) measurement studies, Kim et al. [5] developed a particle characteristics diagnosis system (PCDS) to measure nano-sized particle properties by a combination of particle beam mass spectrometry, scanning electron microscopy (SEM), and energy dispersive x-ray spectroscopy (EDS) [5]. Amaral et al. [6] presents an overview of instruments available on the market for measurement of particulate matter. The main instruments and methods of measuring concentration (gravimetric, optical, and microbalance) and size distribution scanning mobility particle sizer (SMPS), electrical low pressure impactor (ELPI) and others were described and compared. In general, particle detection approaches includes following major approaches [7]: firstly, optical detection approaches are based on detection of intensity of light scattering using single or multiple wavelengths of light. Optical detection efficiency depends on wavelength of light illumination, refractive index of particle, size of particle, sizing volume of the counter, etc. However, optical counters are not suitable for sub$20 \mathrm{~nm}$ particle detection as the smaller particles just do not scatter enough light. This technique works in atmosphere or vacuum conditions. Other options for sub-20 nm particle detection are electrical and condensation based detectors. The second approach is detection based on particle charge where known charge distribution is imparted to particles in an aerosol and these charged particles are attracted towards a detector which measures current and then this current gives a measure of concentration of particles in the aerosol based on the formula below

$$
\mathrm{N}=\frac{I}{e \times N_{p} \times q_{e}}
$$

where, $\mathrm{N}=$ particle number concentration (particles/cc); $e=$ elementary unit of charge, 1.602 x $10^{-19}$ Coulombs; $N_{p}=$ number of charges per particle; $q_{e}=$ flow rate $(\mathrm{cc} / \mathrm{sec}) ; I=$ electrical current (Amps). This methodology needs high concentration and atmospheric pressure to efficiently detect particles. In the third approach, condensation based particle detection, it uses condenses solvent on all particles which grow into droplets as big as 11 microns, and these droplets are then detected easily by an optical particle counter. This method is ideal for sub-20 nm particle detection at high efficiency. However, it cannot be used in low pressure systems and it is very pressure and flow sensitive.

\section{EXPERIMENTAL SETUP}

\subsection{Methodologies of Micro/Nano Aerosols Flow Measurement}

There are several instruments for measuring different characteristics of particulate matter (PM) [6]. The most important measurements of particles are particle concentration and particle size. Particles of varying size, mass, and morphology are often characterised by one of two equivalent size concepts, mobility diameter or aerodynamic diameter. The quantity linking these two size concepts is the (effective) density of the particles. A closely related quantity is the dynamic shape factor of particles, describing the effect of particle shape on the effective 
density. The effective density together with either one of the equivalent diameters is sufficient to predict mechanical dynamics of particles, essential in predicting particle behaviour in the atmosphere, in the human respiratory path, in air cleaning devices, and in measurement instruments. Particle effective density can be found if one of the following combinations is known: mobility size-aerodynamic size, mobility size-particle mass, or aerodynamic size-particle mass [8]. The analyses of particle properties, such as size and component, are required to fully understand the origins of contamination. These allow us to prevent particle generation and to eliminate the particles efficiently [5]. A scanning mobility particle sizer (SMPS) has been developed to measure the size of nano-sized particles in real time, but the measurement can be carried out only at atmospheric pressure. Although prior results improve the performance of a low-pressure differential mobility analyser (DMA), the measurement of the analyzer is still limited to the range of $10-10^{-1}$ Torr. Since most of the fabrication processes for semiconductors require a high-vacuum condition, an in situ monitoring particle beam mass spectrometer (PBMS) designed to measure the particle size distribution at a low pressure (lower than $10^{-1}$ Torr) was developed [5]. In the SMPS ${ }^{\mathrm{TM}}$ spectrometer, sizing is a discreet technique in which number concentrations are measured directly without assuming the shape of the particle size distribution. The method is independent of the refractive index of the particle or fluid, and has a high degree of absolute sizing accuracy and measurement repeatability [9].

\subsection{Experimental Apparatus}

The PM measurement system developed in this study includes four functions: The first part is the cleaning system, the second part is the vacuum pumping system, the third part is the detection system and the fourth part is the sampling device of wafer pollution analysis. Figure 1 shows the schematics of this system. The vacuum chambers used in the experiment are made of SUS304 material and the surfaces are subjected to a standard cleaning procedure to ensure

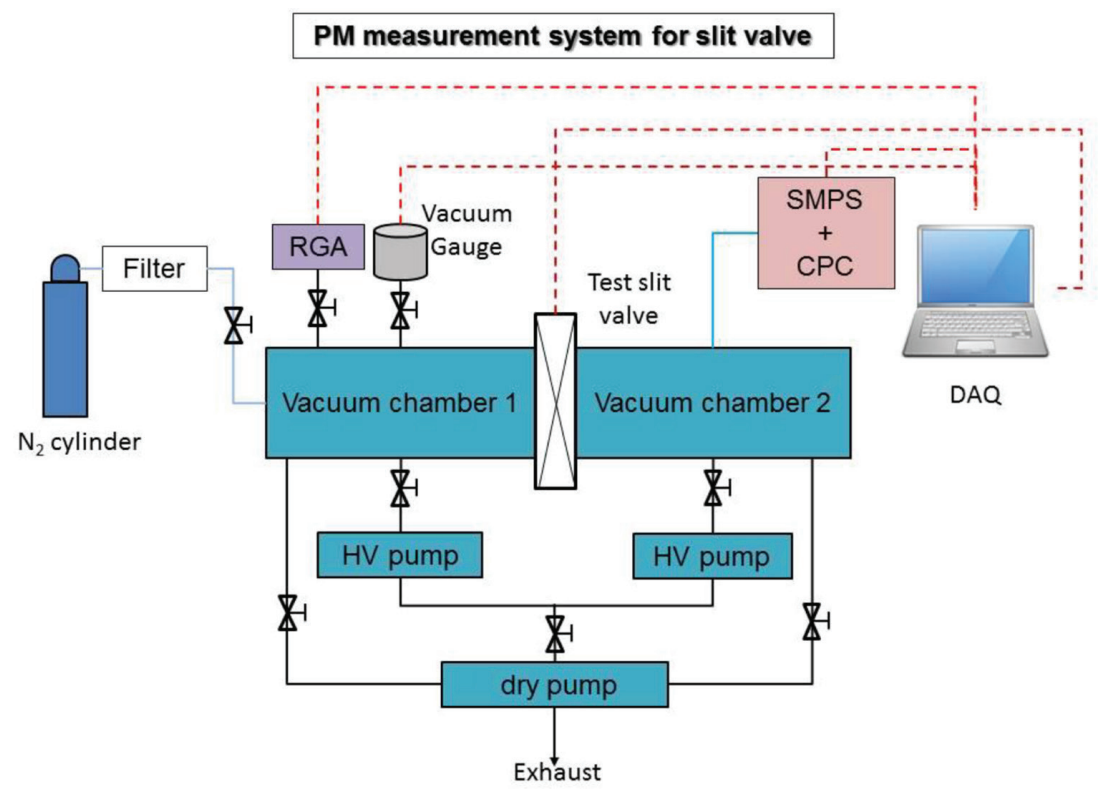

Figure 1: Schematics of the PM measurement system for testing of slit valve in operation. 
its cleanliness. This vacuum system uses a combination pumping system of turbo vacuum pump and dry pump to ensure that no oil and gas pollutions during measurements. The turbo vacuum pumps are with functions of: pumping speed $<920 \mathrm{~Pa}$.L/s, ultimate pressure $<1 \mathrm{E}-6$ $\mathrm{Pa}(<7.5 \mathrm{E}-9$ Torr), with grease lubricated ball bearing; and the dry vacuum pump is with functions of: pumping speed $<500 \mathrm{~L} / \mathrm{min}$, ultimate pressure $<0.5 \mathrm{~Pa}, \mathrm{KF} 40$ flange connection. The cold cathode vacuum gauge with measurement ranges of 1 x 10 E-9 to 1 x 10 E-2 mbar $(0.75 \times 10$ E- 9 to $0.75 \times 10$ E-2 Torr) and the vacuum gauge by MEMS-Pirani technology with measurement range of $5.0 \times 10^{-5}$ mbar to atmosphere ( $3.75 \times 10^{-5}$ Torr to atmosphere) are used to monitor the vacuum pressures in each chambers for the high- and low-vacuum ranges, respectively. The slit valve under test is with functional specifications of $50 \times 336 \mathrm{~mm}$ in size, sealing material for the FKM material, the maximum leakage rate $<1.0 \mathrm{E}-9$ Torr.L/s (He), etc. Before the test, the testing vacuum chambers are purging using $\mathrm{N}_{2}$ to a certain level of cleanliness, and the four-quadruples residual gas analyzer (RGA) with the basic function of $0 \sim 100$ amu, and maximum operating pressure $<1 \mathrm{E}-5$ Torr (5E-4 mbar) is used to check the residues in vacuum chambers. For each experiment requires venting to the atmosphere, the $\mathrm{N}_{2}$ gas (99.9999\% purity) is supplying from a cylinder through the filter and then conduct into the vacuum chambers.

\subsection{Particles Concentration Measurements}

Concentration measurements by optical particle counter (OPC) and condensation particle counter (CPC) are two methods often used commonly in a variety of applications. In OPC, detection of scattered light intensity by particles can be used to measure the particle size and the number of particles, and the detectable particle size ranging from 0.3 to 20 microns. The CPC is mainly used to mix the particles with saturated vapors (isopropanol, n-butanol, water), and then pass the mixed gas through a region of low temperature to produce supersaturated conditions so that the vapor condenses on the surface of the particles. The particles thus grow to a detectable range ( $\sim 12$ microns), and then use the photodetector to measure the number of particles; particle size in range of 0.005 to 1.0 microns can be measured. For a CPC system, it is consisting of three parts: the first part is the flow control system, the second part is the detector and the third part is the optical signal microprocessor. For the detector, it contains saturators, condensers and optical systems, etc. As to the theory of the condenser, its working principle is based on the saturation ratio which is defined by the ratio of the vapor molecules produced under the vapor pressure $(\mathrm{P})$ to the saturated vapor pressure (Ps) under the same temperature. At a certain saturation ratio, the vapor molecules will be condense to the larger size particles only, and this threshold is called the Kelvin diameter and its relationship with saturation ratio is as following

$$
\text { Saturation ratio }=\frac{P}{P_{S}}=e^{\frac{4 \gamma M}{\rho R T D}}
$$

where $\gamma=$ surface tension of condensate, $M=$ molecular weight of condensate, $\rho=$ density of condensate, $R=$ ideal gas constant, $T=$ absolute temperature, $D=$ Kelvin diameter. The particle counting efficiency which is defined as

$$
\frac{N_{0}}{N_{t}}=e^{-N_{t} Q \tau}
$$


where $N_{t}=$ real particle counts, $N_{0}=$ instrument particle counts, $Q=$ flow rates and $\tau=$ time period of particle through light. The CPC system in this study is with specifications: minimum detectable diameter (D50) of $2.5 \mathrm{~nm}$, concentration range in 0 to 4E6 particles $/ \mathrm{cm}^{3}$ (accuracy $\pm 10 \%$ to 400,000 particles $/ \mathrm{cm}^{3}$ ), flow rate in high range $1.5 \pm 0.15 \mathrm{~L} / \mathrm{min}$ (response time $\sim 250 \mu \mathrm{s}$ ) and in low range $0.6 \pm 0.006 \mathrm{~L} / \mathrm{min}$ (response time $\sim 400 \mu \mathrm{s}$ ), etc.

\subsection{Particles Size Measurements}

Typically, the size concentration of nanoparticles in the environment is measured by SMPS or ELPI. Measurement methodology of SMPS is mainly utilize the principle of different sizes of particles will have different electrical mobility, so that the particles are charged by neutralizer firstly, and after that particles with different sizes are separated by DMA. Finally, particle counts with different particle sizes and size concentrations can be obtained by CPC. Basic principle of ELPI is to make the particles through the corona charger, after charging, the particles are directed into the low-voltage impactor. When the charged particles are impacted onto the substrate, the number of particles in different particle sizes can be known by measuring the electricity. In SMPS, the charged particles with different sizes are separated by the DMA. Working principle of the DMA is by the metal cylinder and the center of the metal rods formed, when the charged particles into the charged metal cylinder, and, in the electric field, the Coulomb force will make it moves to different distances, as shown in Fig. 2. In this study, the DMA system is with specifications: particle sizes in the range of $2.5 \mathrm{~nm}$ to $150 \mathrm{~nm}$, size concentration in the range of 0 to $1 \mathrm{E} 7$ particles $/ \mathrm{cm}^{3}$, and water as working fluid, etc.

\subsection{Wafer Contaminants Measurements}

A SEM uses a focused beam of high-energy electrons to generate a variety of signals at the surface of solid specimens. The signals that derive from electron-sample interactions reveal information about the sample including external morphology (texture), chemical composition, and crystalline structure and orientation of materials making up the sample. It is used widely in metallurgy, geology, biology and medicine, to name just a few. In this study, the Hitachi S-4300 SEM system is used and its basic functions are 5nm resolution, 20X 25,000X in magnification, 1E-4 Pa in vacuum (sample chamber). Atomic force microscopy (AFM) is a type of scanning probe microscopy where the probe can be used to physically contact the substrate to obtain topographical information as well as material properties. The resolution of an atomic force microscope is limited primarily by the radius of the tip of the cantilever (collectively known as probe), the sensitivity of the detection system, cantilever spring constant, and noise. In this study, the Digital Instrument model 3100 AFM system is used to detect the particles on witness wafer. It's basic functions include $100 \mathrm{~mm}$ x $125 \mathrm{~mm}$ viewing range,

\section{SMPS Theory of operation}

\begin{tabular}{|c|c|c|c|c|}
\hline Aerosol & Impactor & Neutralizer & DMA & $\mathrm{CPC}$ \\
\hline & $\begin{array}{c}\text { Sample } \\
\text { conditioner }\end{array}$ & $\begin{array}{c}\text { Charge } \\
\text { conditioner }\end{array}$ & $\begin{array}{c}\text { Size } \\
\text { selector }\end{array}$ & Counter \\
\hline
\end{tabular}

Figure 2: Theory of operation of a SMPS system. 
travel distances of $\mathrm{x}, \mathrm{y}, \mathrm{z}$ axes are $100 \mathrm{~mm}, 100 \mathrm{~mm}, 4 \mathrm{~mm}$, respectively. An EDS detector is used to separate the characteristic x-rays of different elements into an energy spectrum, and EDS system software is used to analyze the energy spectrum in order to determine the abundance of specific elements. EDS can be used to find the chemical composition of materials down to a spot size of a few microns, and to create element composition maps over a much broader raster area. Together, these capabilities provide fundamental compositional information for a wide variety of materials. In this study, the Oxford INCA system is used to inspect the composition of particles on witness wafer.

\section{RESULTS AND DISCUSSION}

In this paper, the particle generating behaviour while the testing slit valve is in operation is experimentally investigated. Experimental methods include CPC, SMPS, SEM, EDS and AFM. Due to the vacuum environment limitations, some measurements must be conducted in atmosphere. Therefore, as shown in Fig. 3, this study will be divided into two topics: the first topic is specific for the PM measurement and analysis in atmospheric environment and the second topic is discussion for the vacuum environment.

While a slit valve is periodically actuating the open and close motion between two vacuum chambers, many factors like materials of slit valve body and O-ring, manufacturing quality of part surfaces, cycle time, lifetime usage and the operating environment etc. may incur particle generation in vacuum chamber which may be attributed to one of the reason of wafer contaminations. This study designed a vacuum system and setup appropriate instruments to investigate the PM measurement of an innovative designed slit valve. This vacuum system is designed to easily replace different slit valves and its internal components and to control the periodically cycle numbers and cycle times for slit valve testing. Besides, this system can be used to detect the particle sizes and numbers inside the vacuum chambers or under atmospheric condition and to explore the particle size distributions. Furthermore, by specific allocated witness wafers in vacuum chambers, compositions of particles can be investigated. Before experiments, a SOP is conducted routinely to study the background pressures and to continuously record pressure variations of this vacuum system. This measurement system uses the pumping system by connecting turbopumps with dry pumps to pump the system to high vacuum status. As shown in Fig. 4, after 7 hours pumping, the ultimate vacuum pressure in this system can reach the requirement of $<1 \mathrm{E}-5$ Torr. Each pumping experiment is repeated three times to insure the minimum experimental uncertainty.

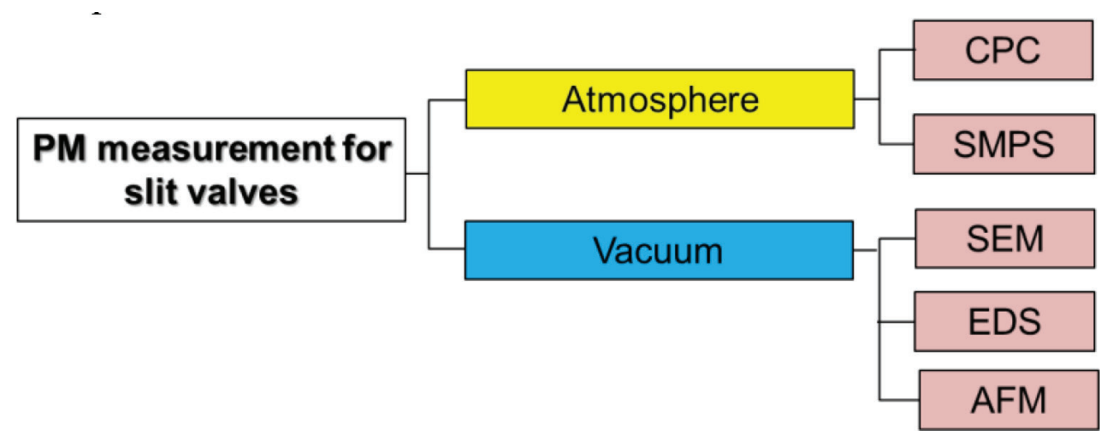

Figure 3: Testing classifications of the PM measurement system for slit valve in operation. 


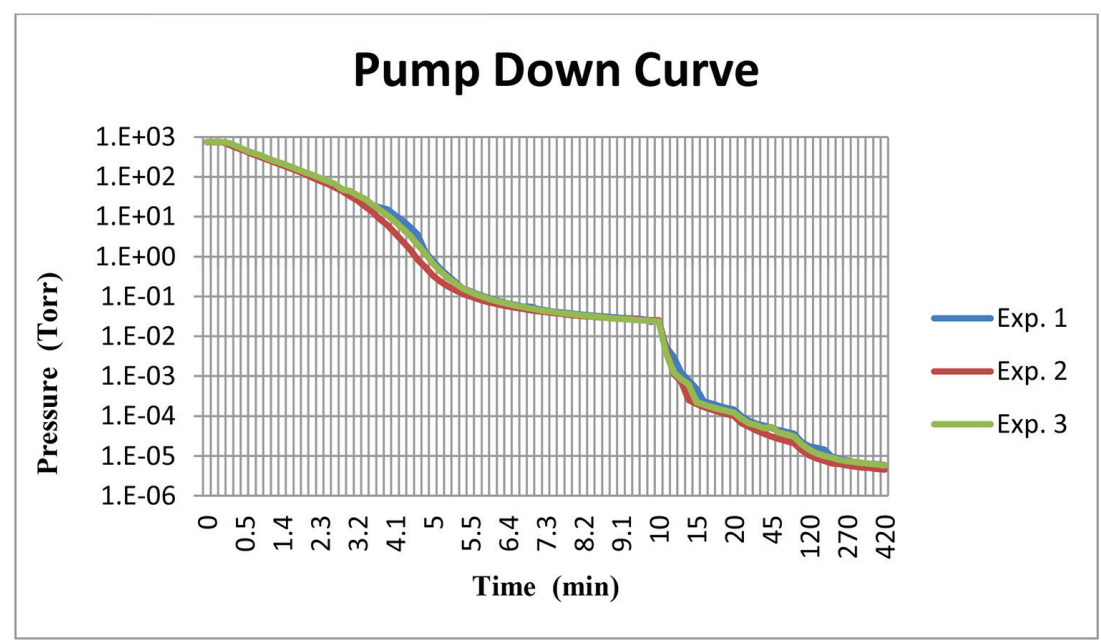

Figure 4: Background pressure of the PM measurement system for slit valve testing.

\subsection{PM Measurements in Atmosphere}

For experiments in atmospheric conditions, it is mainly to measure particle sizes and concentrations in vacuum chamber which may attribute to particles generated by the end of periodically operating a slit valve. In this experiment, the particle sizes and size distributions are measured by CPC and SMPS instruments and the gathered results are compared to the measured particle sizes by SEM and AFM and are used to evaluate the assumptions of particle generating sources and mechanisms. As shown in Fig. 5, follow this procedure, the same

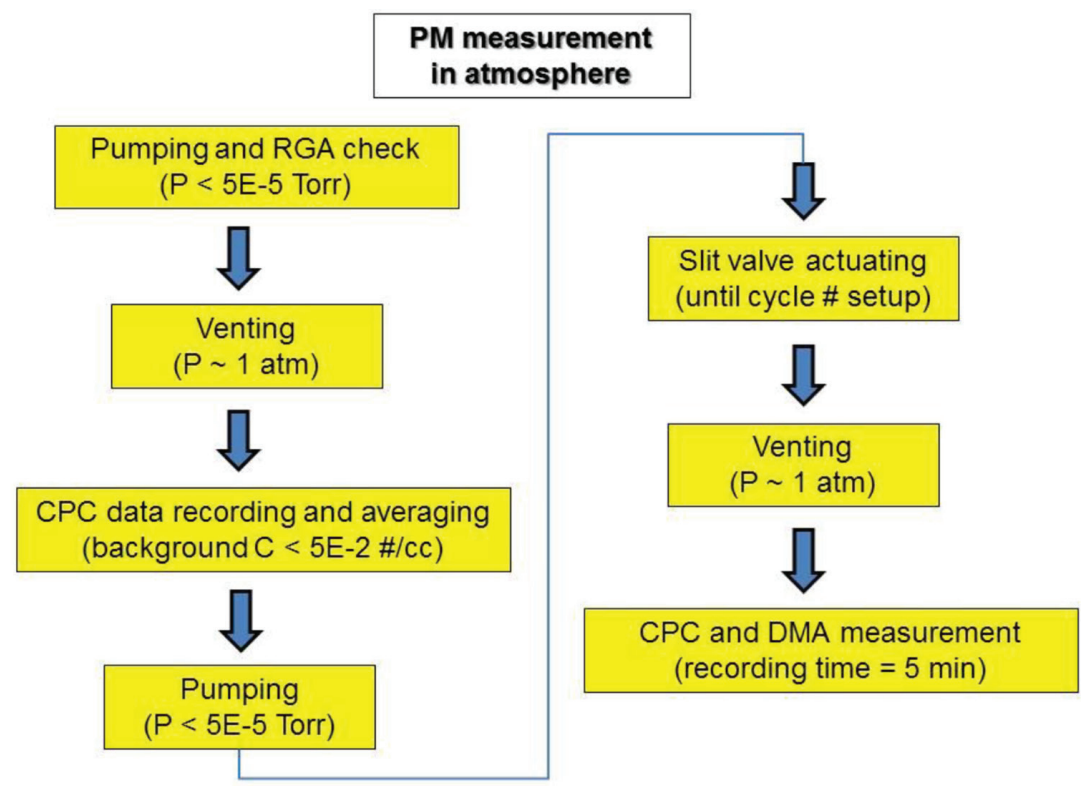

Figure 5: Procedures of PM measurements in atmosphere for a slit valve. 
slit valve is tested for 10,000, 20,000 and 40,000 cycles respectively in vacuum then particle sizes and concentrations are measured by CPC and DMA methods. Figure 6 shows the particle concentrations generated and stored in vacuum chambers while this slit valve actuated $10,000,20,000$ and 40,000 cycles, respectively. It can be found experimentally that the particle sizes and particle concentrations increase as the number of motions increases, as shown in Table 1.

\subsection{PM Measurements in Vacuum}

Figure 7 shows the experimental procedures of PM measurements under vacuum conditions. This experiment was conducted by placing totally eight 2 inch wafers in the chamber 1 and chamber 2, and then performing the slit valve open/close testing cycles under vacuum by the end of setup cycle numbers to understand the deposition of particles on the wafer surface. After the test, the wafer is taken out and placed in the FOUP like box then the particle sizes of the deposited particles on wafer is measured by SEM and AFM. The results are compared with the SMPS measurement. In this study, cycle numbers 20,000 and 40,000 respectively are tested and data are analysed. As shown in Fig. 8, this AFM measurements are based upon

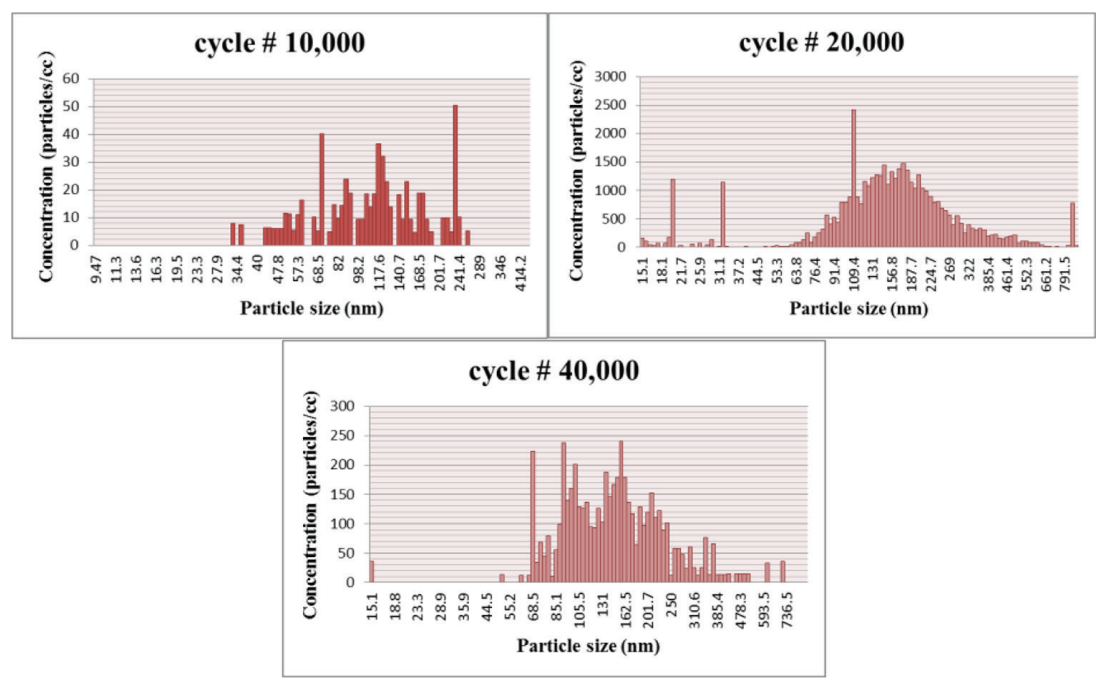

Figure 6: Concentrations in vacuum chamber after slit valve actuated 10,000, 20,000 and 40,000 cycles.

Table 1: Averaged particle concentrations and diameters in different cycle numbers testing of a slit valve.

\begin{tabular}{llll}
\hline & \multicolumn{2}{l}{ Cycle numbers } & \\
\cline { 2 - 4 } & 10,000 & 20,000 & 40,000 \\
\hline Mean (counts $\left./ \mathrm{cm}^{3}, \pm 10 \%\right)$ & 13.9 & 417.3 & 559.0 \\
Mean (nm, $\pm 1 \% @ 100 \mathrm{~nm})$ & 113.2 & 155.0 & 171.2 \\
\hline
\end{tabular}




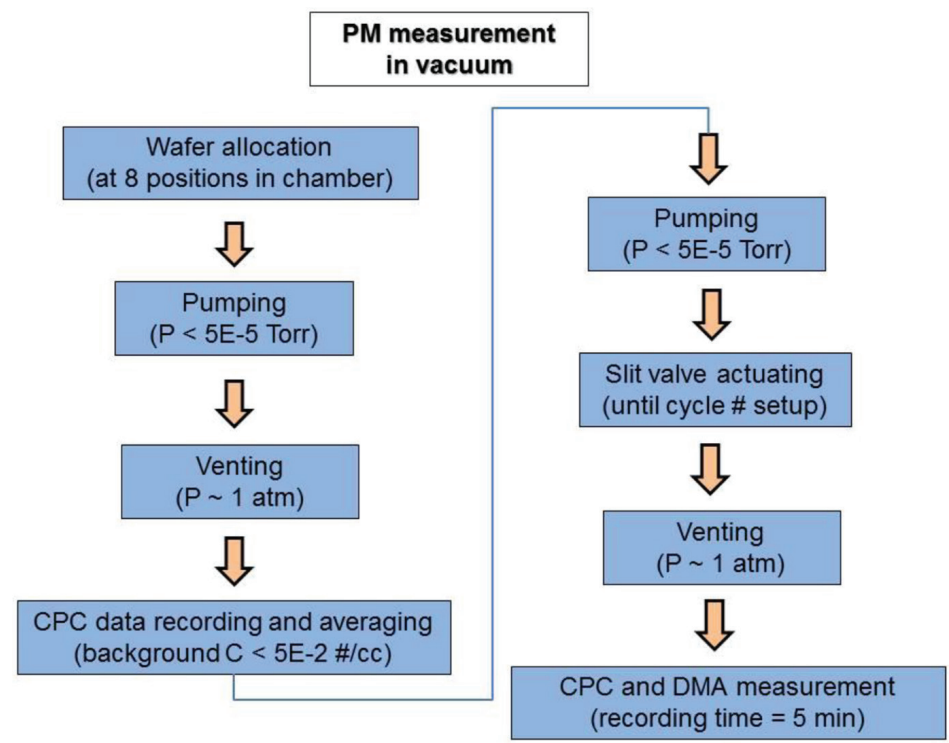

Figure 7: Procedures of PM measurements in vacuum for a slit valve.

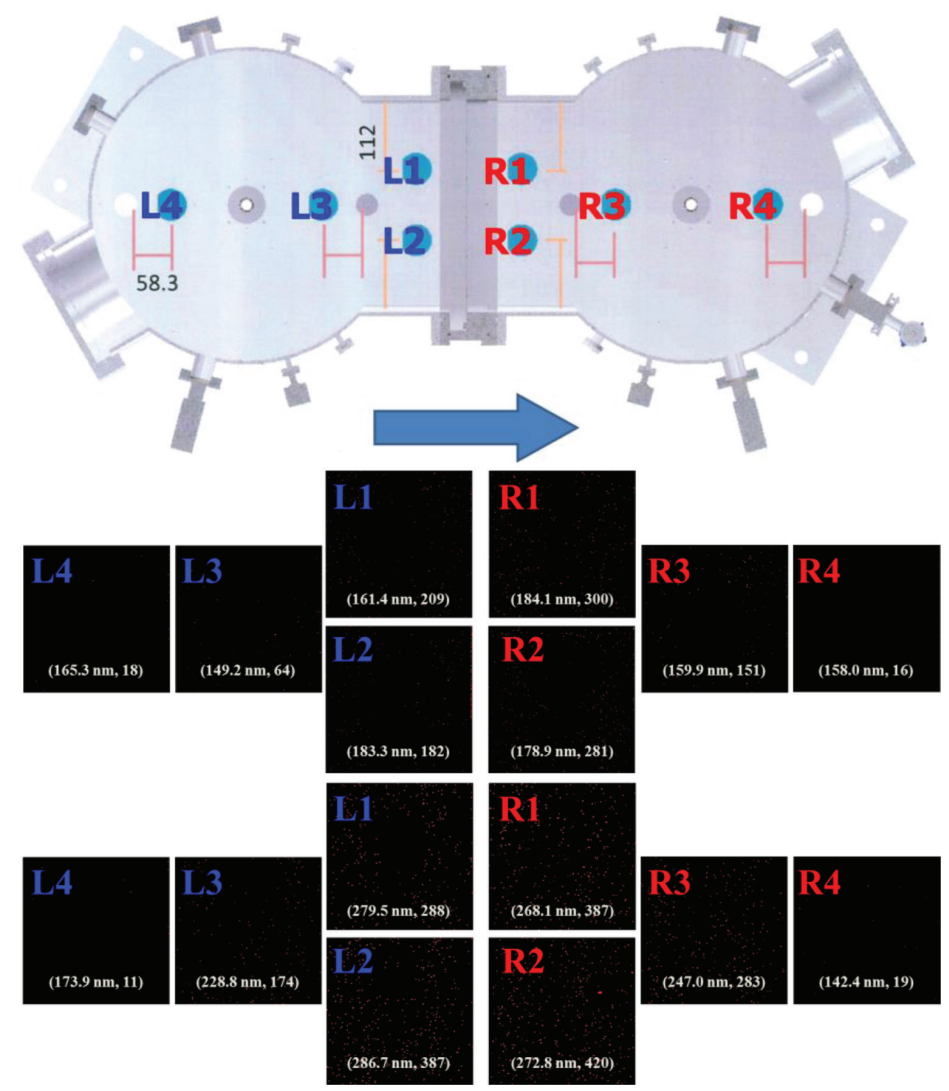

Figure 8: AFM measurements for witness wafer after slit valve operated 20,000 (middle) and 40,000 (down) cycles, respectively. 
Table 2: Comparison of averaged particle diameters measured by SMPS and AFM.

\begin{tabular}{lllll}
\hline & 20,000 cycle numbers & \multicolumn{3}{l}{40,000 cycle numbers } \\
\cline { 2 - 5 } & SMPS & AFM & SMPS & AFM \\
\hline Mean $(\mathrm{nm})$ & $155.0 \pm 1 \% @ 100 \mathrm{~nm}$ & $184 \pm 2 \%$ & $171.2 \pm 1 \% @ 100 \mathrm{~nm}$ & $286.7 \pm 2 \%$ \\
\hline
\end{tabular}

the basic condition that the bare wafer has less than 10 particles which is greater than $0.5 \mu \mathrm{m}$. The experimental results, Table 2, show that differences between SMPS and AFM measurements are about $15.7 \%$ and $40.3 \%$, respectively. The detection methods of SMPS and AFM are different as described in previous sections; therefore, these values are just for reference and need to improve in future study.

\section{CONCLUSIONS}

This study designed a vacuum system and setup a series of instruments to investigate the PM measurement of an innovative designed slit valve with the size $(50$ x $336 \mathrm{~mm})$. In atmosphere experiments, the particle sizes and size distributions are measured by CPC and SMPS instruments and the gathered results are compared to the measured particle sizes by SEM and AFM and are used to evaluate the assumptions of particle generating sources and mechanisms. Experimental results show that the particle sizes and particle concentrations increase as the number of motions increases. To examine the particle generations in vacuum, the particle sizes of the deposited particles on wafer are measured by SEM and AFM. The results are compared with the SMPS measurement. In this study, cycle numbers 20,000 and 40,000, respectively, are tested and data are analysed. Experimental results show that differences between SMPS and AFM measurements are about $15.7 \%$ and $40.3 \%$, respectively. Also, the possible particle generations originate from O-ring abrasion during operating in vacuum environment and compositions of O-ring materials and particles deposited on the witness wafers are inspected by the EDS instrument. No enough data to conclude the reasons for sources of generated particles in this method.

\section{ACKNOWLEDGEMENTS}

This work was supported by the Ministry of Science and Technology under the contract numbers of MOST 104-2622-E-492-024 -CC2 and MOST 105-2622-E-492-028 -CC2.

\section{REFERENCES}

[1] Hoffman, M., Singh, B. \& Thomas III, J. H., Handbook of Vacuum Science and Technology, Elsevier Inc. 1998.

[2] Striving towards zero particle vacuum valves, current and future defectivity issues from components in the semiconductor industry, Albany, NY, November 12, 2012, available at: www.sematech.org/meetings/archives/defectivity/10349/index.htm. (accessed 23 February, 2017).

[3] VAT company brochure. available at: www.vatvalve.com/business/valves/catalog. (accessed 23 February, 2017).

[4] Sonderegger, K., Dür, M., Buthig, J., Pantazis, S. \& Jousten, K., Very fast-opening UHV gate valve. Journal of Vacuum Science and Technology A, 31(6), p. 060601, 2013. https://doi.org/10.1116/1.4813836 
[5] Kim, D., Mun, J., Kim, H.U, Yun, J-Y., Kim, Y-J., Kim, T., Kim, T. \& Kang, S-W., Development of particle characteristics diagnosis system for nanoparticle analysis in vacuum. Review of Scientific Instruments, 87, p. 023304, 2016. https://doi.org/10.1063/1.4942247

[6] Amaral, S.S., Carvalho, J.A., Martins Costa, M.A. \& Pinheiro, C., An overview of particulate matter measurement instruments. Atmosphere, 6, pp. 1327-1345, 2015. https://doi.org/10.3390/atmos6091327

[7] Progress and challenges in sub $20 \mathrm{~nm}$ particle detection from vacuum components, Current and Future Defectivity Issues from Components in the Semiconductor Industry, Albany, NY, November 12, 2012, available at: www.sematech.org/meetings/archives/ defectivity/10349/index.htm. (accessed 23 February, 2017).

[8] Ristimaki, J., Virtanen, A., Marjamaki, M., Rostedt, A. \& Keskinen, J., On-line measurement of size distribution and effective density of submicron aerosol particles. Journal of Aerosol Science, 33, pp. 1541-1557, 2002. https://doi.org/10.1016/s0021-8502(02)00106-4

[9] Scanning Mobility Particle Sizer Spectrometer 3936, available at: www.tsi.com/scanning-mobility-particle-sizer-spectrometer-3936/ (accessed 23 February, 2017). 